\title{
Optical Characteristics of Young Quasars as Sources of the Cosmic X-Ray Background
}

E. Boldt and D. Leiter

JULY 1983

Natıonal Aeronautıcs and

Space Administration 
OPTICAL CHARACTERISTICS OF YOUNG QUASARS

AS SOURCES OF THE COSMIC X-RAY BACKGROUND

\author{
Elihu boldt and Darryl Leiter ${ }^{1}$ \\ Laboratory for High Energy Astrophysics \\ NASA/Goddard Space Flight Center \\ Greenbelt, Maryland 20771
}

ABSTRACT

The sources which dominate the thermal cosmic X-ray background cannot have $X$-ray spectra similar to the power laws measured for bright active galactic nuclei. We pursue the optical consequences of this disparity by considering a standard model for the photoexcitation and heating of the line emitting gas surrounding a central source (e.g. such as a quasar). The optical line emission to be associated with compact young quasar sources having the same $X$-ray spectrum as the $X$-ray background is found to be substantially different from that characteristic of typical quasars. Implications on quasar source counts and the identification of such new objects are discussed.

Subject headings: cosmology - galaxies: nuclei - quasars $X$-rays: sources - X-rays: spectra

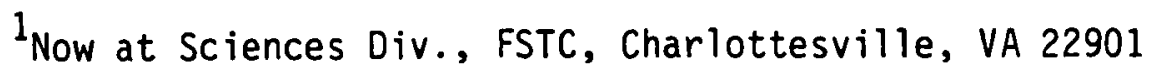




\section{INTRODUCTION}

In considering the possibility that young quasars and/or other compact objects at high redshifts $(z \geq 3)$ constitute most of the $X$-ray background (Leiter and Boldt 1982, Paper I) we need to confront the:following observational conclusions:

1) Most of the cosmic $X$-ray background (CXB) arises from apparently thermal type emission characterized by $k T \leqslant 30(1+z) \mathrm{keV}$ (Paper I, Appendix D). 2) If point sources dominate the $C X B$, then they number more than optically bright quasars (Paper I, Appendix C, p. 15).

Assuming that the principal sources of the CXR are indeed compact objects related to quasars, we identify some of the key optical signatures characteristic of the spectral evolution that could be inferred from these observations. In particular, optical line intensities calculated for such compact sources of the CXB are compared to the intensities similarly calculated for typical quasars.

II. SPECTRUM OF THE RESIDUAL COSMIC X-RAY BACKGROUND

The sources which dominate the cosmic $X$-ray background (CXB) over the spectral range of maximum flux (i.e. from - $3 \mathrm{keV}$ to $\geq 50 \mathrm{keV}$ ) can not have spectra similar to the power laws measured for bright active galactic nuclei (AGN) over this same band (Boldt 1981; nezotti et al. 1982; Paper 1). In fact, the spectrum of the total CXB over this band as observed with HEAO-1 may be well described in terms of optically thin thermal bremsstrahlung (Marshall et al. 1980) at $k T \approx 40 \mathrm{keV}$. Furthermore, since a significant portion of the C.XB must arise from usual AGN, even the composite spectrum from those sources which actually do dominate the CXB cannot be identical to that of the total CXR. It is evident that the spectrum of the composite $X$-radiation from those sources which dominate the CXB must be obtained from the total CXB spectrum by 
subtracting the estimated contributions of known source populations, such as usual AGN (including bright quasars) and clusters of galaxies. To estimate the foreground spectrum due to the composite of such sources we use the formulation described in Paper I (see Appendix D, considering the case for $\gamma=$ 0.9, $B=0$ ). When subtracted from the "total $C \times B$ " spectrum observed with HEAO-1, this yields a first approximation of the spectrum to be associated with the "residual CXB".

Figure 1 exhibits the ratio of our approximation of the residual CXB to the total CXB observed with $H E A O-1$ as a function of the photon energy (hv). Over the band $h v \approx(10-30) \mathrm{keV}$, this ratio exhibits a flat peak corresponding to a residual $C X B$ that is $\geqslant 3 / 4$ of the total $C X B$; at hv $\geqslant 100 \mathrm{keV}$ we expect that the residual CXB will fall to $<1 / 2$ of the total CXB (Rothschild et al. 1983). At hv < $10 \mathrm{keV}$, the residual CXB spectrum is clearly harder than that of the total $C X B$; it is this rather pronounced effect that we focus upon in this paper.

We note that the spectrum for optically thin thermal bremsstrahlung, as described by Maxon (1972), may be well approximated (over the band 3-100 keV) by the form

$$
\frac{d S}{d(h v)} \propto(h v)^{-\alpha} \exp [-h v / B]
$$

where $\alpha=0.19$ for $k T=B=200 \mathrm{keV}$ and $\alpha \approx 0.3$ for $k T=B=40 \mathrm{keV}$. In general the thermal $X$-radiation from clusters of galaxies (where $k T<10 \mathrm{keV}$ ) requires $\alpha \geq 0.4$. The power-law X-ray spectra for $A G N$, in the representation corresponding to hv $<B$ (Rothschild et al. 1983), have $\alpha \approx 0.7 \pm 0.1$ (see Figure 3 presented by Mushotzky 1982; Petre, Mushotzky and Holt 1983). The residual CXB spectrum may be fitted with this form (eq. (1)) provided $\alpha<0.2$ 
and $B \lesssim 30 \mathrm{keV}$; a good fit is provided by $\alpha=0$, with $B=23 \mathrm{keV}$ (Paper I, App. D).

For optically thin thermal bremsstrahlung, the requirement that $\alpha<0.2$ for the residual CXB (as viewed by the observer) implies $k T \gtrsim 200 \mathrm{keV}$ at the sources. Under the condition $B \lesssim 30 \mathrm{keV}$ for the observed redshifted spectrum, these sources would have to be located at $z \gtrsim 6$ (i.e. Well beyond any known quasar). However, if such sources are compact objects radiating close to the Eddington luminosity limit (Paper I) then the possibility of a pronounced spectral feature corresponding to electron-positron annihilation must be considered (Ramaty and Meszaros 1981; Kazanas and Shafer 1983). The fact that such a feature is not observed in the CXB (Kanzanas and Shafer 1983) implies that the thermal emission to be associated with compact sources of the CXB radiating close to the Eddington limit could not be due to thin bremsstrahiung alone. For a compact thermal source involving Comptonization, on the other hand, this line is suppressed (Kanzanas and Shafer 1982). Furthermore, Comptonization lowers the value of $\alpha$, relative to that for thin bremsstrahlung at the same temperature (Mckinley and Ramaty 1983), thereby allowing kT $<200$ keV (and $z<\overline{0}$ ) for major sources of the CXB (e.g. such as those considered in Paper I). A suitable range of values for the spectral index (i.e. $0.2>\alpha \gtrsim 0)$ is readily obtained via Comptonization if the electron scattering optical depth is somewhat greater than unity but not so large as to produce a discernible Wien peak (Pozdnyakov, Sobol and Syunyaev 1977; Leiter 1983). In any event, we emphasize that the $X$-ray spectra characteristic of the sources that dominate the CXB must be remarkably flat (below $\sim 10 \mathrm{keV}$ ), relative to most bright $A G N$, and it is the optical consequences of this disparity that we pursue here. In particular, we examine the case where this flat spectrum extrapolates to the UV, such as expected for an isothermal source that is not too optically thick. 
III. A CALCULATION OF YOUNG QUASAR OPTICAL CHARACTERISTICS

In considering the central UV source responsible for the photoexcitation of the line emitting gas associated with typical quasars (QSOS), the continuum spectrum has been taken as a power-law of spectral index in the range $\alpha \approx 0.5$ - 1 (Netzer, Wills and Wills 1982), consistent with the indices noted in the $X$-ray band for Seyfert galaxies (Mushotzky 1982) and the values

of $\alpha_{\mathrm{R}}^{0}$ characterizing the ratio of optical-to-radio emissions for strong compact radio-selected quasars (Condon, Jauncey and Wright 1978). This is in sharp contrast to those sources that dominate the $C \times B$, where we expect $\alpha \approx 0$ (see Section II). The optical emission from gas photoionized by a source of UV and X-ray photons with such a flat continuum spectrum could be substantially less than that of typical QSOS, where the ionizing continuum is much softer. If young quasars (YQSOS) are indeed representative of the dominant sources of the $C X B$, this would imply a strong evolution in the optical characteristics of OSOS as they emerge from the YQSO stage.

The central source to be associated with a typical quasar could have a substantial optically thick thermal UV component in addition to a power-law continuum (Malkan and Sargent 1982). Although such thermal emission would have to reside mainly below the Lyman limit, at wavelengths somewhat longer than $0.1 \mu$ (Green et a1. 1980), it would imply that the overall power-1aw component for many quasars could be even steeper than sometimes inferred (i.e., with $\alpha \approx 1$ a closer estimate than $\alpha \approx 1 / 2$; Malkan and Sargent 1982). Moreover, that portion of any thermal UV emission just beyond the Lyman limit would have the effect of further softening the composite spectrum for the ionizing continuum, relative to that of the power-law component alone. We assume that any such thermal UV component (at $T \leq 10^{5} \mathrm{~K}$ ) present for QSOS is essentially absent in the earlier much harder thermal emission characteristic 
of Yonsos. This is compatible with the scenario in which the non-thermal component of QSO emission is generated at the expense of cooling the hot inner region of the accretion disk dynamo initiated in the YQSO phase (Paper I).

In order to compare the optical emission to be expected from those sources that dominate the $C X B$ (e.g. young quasars) with that already observed from quasars, we consider a standard picture in which a central source of continuum radiation is surrounded by a spherical shell of gas, taken here as an idealization of the physical clouds associated with AGN line emitting regions (Davidson and Netzer 1979). Photoionization models are characterized by 1) the spectrum and luminosity of the central continuum source, 2) the density and chemical composition of the surrounding gas, 3) the distance between the central source and the gas and, for the case of a hard $x$-ray continuum (Ferland and Mushotzky 1982), 4) the thickness of the gas shell. Calculations are performed with the program described by Ferland and Truran (1981). We follow closely the procedures used by Ferland and Mushotzky (1982) in their analysis of NGC 4151 and employ the same "solar" chemical composition for the gas. The continuum spectrum of the central source over the band $13.6 \mathrm{eV}-10^{5} \mathrm{eV}$ is taken as

$$
\frac{d l}{d h v} \propto(h v)^{-\alpha}
$$

The luminosity of this source $\left(L \mathrm{erg} \mathrm{s} \mathrm{s}^{-1}\right)$ is evaluated over the same band. The mean photon energy $\langle h \nu(\alpha)\rangle$ over this continuum is a function of the spectral index $\alpha$, as follows

$$
\begin{aligned}
& \langle h \nu(\alpha=0)\rangle=1.79 \times 10^{-8} \text { erg }+(11.2 \mathrm{keV}) \\
& \langle h \nu(\alpha=0.5)\rangle=1.87 \times 10^{-9} \mathrm{erg}+(1.17 \mathrm{keV})
\end{aligned}
$$




$$
\langle h \nu(\alpha=1.0)\rangle=1.94 \times 10^{-10} \mathrm{erg} \rightarrow(0.121 \mathrm{keV})
$$

For very steep spectra (i.e. $\alpha \gg 1$ ) the average photon energy approaches the Rydberg $\left(E_{\mathrm{R}} \equiv 2.18 \times 10^{-11} \mathrm{erg} \rightarrow 13.6 \mathrm{eV}\right)$.

The photoexcitation model is further specified by the hydrogen number density ${ }^{1}$ of the gas $\left(n_{H}\right)$ and the ionization parameter $(U)$, given by

$$
u \equiv n_{v} / n_{H}
$$

where $\left(n_{v}\right)$ is the number density of photons impinging upon the inner surface of the gas shell. We assume that the density within the shell is constant. Although constant pressure models are probably more appropriate, the assumption of constant density allows a more meaningful comparison between situations with different $U$ and is sufficiently accurate for these calculations (Ferland and Mushotzky 1982). The rate of photon emission by the central source $\left(Q \mathrm{sec}^{-1}\right)$ is related to $U$ and $n_{H}$ in terms of the inner radius $(r)$ of the shell, as follows

$$
Q \equiv L /\langle h v\rangle=4 \pi r^{2} c n_{H} U
$$

where $(c)$ is the speed of light.

The thickness of the gas shell is measured by the hydrogen columnar number density $\left(\mathrm{N} \mathrm{cm}^{-2}\right)$. We relate $\mathrm{N}$ to $U$ by assuming that most incident photons are absorbed, most of the hydrogen is ionized and that there is an equilibrium between ionization and recombination (e.g. see Ferland and Mushotzky 1982). For a recombination coefficient $\propto T^{-3 / 4}$, this yields 


$$
\log (N / U) \approx 23+\frac{3}{4} \log T_{4}
$$

where $T_{4} \equiv T /\left(10^{4} K\right)$. For the cases considered here, we find that $T_{4} \approx 1-10$ whereby equation $(6)$ yields $\log (N / U) \approx 23.00-23.75$. For our calculations we use

$$
\log (N / U)=23.75
$$

With $U \approx 10^{-1}$, equation (7) yields a characteristic shell thickness corresponding to $\sim 1^{-1} \mathrm{~g} \mathrm{~cm}^{-2}$, comparable to that of the clouds inferred for NGC 4151 (Holt et al. 1980). For such clouds, photoelectric absorption can be appreciable for energies up to - $3 \mathrm{keV}$. When the characteristic thickness is $\leq 10^{-2} \mathrm{~g} \mathrm{~cm}^{-2}$ (corresponding to $11 \leq 10^{-2}$ ), however, photoelectric absorption of $X$-rays (hv $\geq 1 \mathrm{keV}$ ) becomes relatively weak.

The spectral steepness of the continuum emission from the central source is a most critical input for the model. This is illustrated in Figure 2, where we exhibit the total emission rate $O(E)$ of photons (E< hv<100 keV) for two different power-law spectra as a function of the energy lower-limit (E) for the integration. Both of these spectra correspond to the same luminosity over the band $13.6 \mathrm{eV}-100 \mathrm{keV}$. For a lower-limit energy comparable to the Rydberg $(E \approx 13.6 \mathrm{eV})$, the 0 for the steep $(\alpha=1)$ power-law exceeds that for the flat $(\alpha=0)$ spectrum by about two orders of magnitude. Not until well into the $X$-ray band ( $E \geqslant 3 \mathrm{keV}$ ) does the $Q$ for the flat spectrum exceed that for the steep power-law. As indicated, unit optical depth $(\tau=1)$ for a $3 \mathrm{keV} X$-ray occurs at a shell thickness $-10^{-1} \mathrm{~g} \mathrm{~cm}^{-2}$. For the steep spectrum, however, most photons exceeding the Rydberg energy reside at hu $<40 \mathrm{eV}$, a regime where photoelectric absorption by hydrogen is assured even when the gas shell is much thinner.

In comparing the two ionizing continua displayed in Figure 2 the main 
effect of interest here is that of the pronounced increase in the output of UV photons with rising spectral steepness $(\alpha=0$ to $\alpha=1)$. This leads to an increase in the production of ions (such as $\mathrm{H}^{+}$) from species with low ionization potentials and results in an enhancement in associated line emissions (e.g. Lya). Although the primary ionization of such species is generally dominated by UV photons, X-ray interactions can make major contributions to secondary ionization and heating. For thick shells $\left(\geqslant 10^{-2} \mathrm{~g}\right.$ $\mathrm{cm}^{-2}$ ), the input of $X$-rays can lead to appreciable line emission from ions of high ionization potential, such as the lithium-like ions $0_{V I}$ and NeVIII From equation (5) and equation (7), we note that the total hydrogen content of the shell $\left(4 \pi r^{2} N\right)$ is specified by $n_{H}$ and $Q$ as follows.

$$
\log \left(4 \pi r^{2} N\right)=13.27+\log (Q)-\log \left(n_{H}\right)
$$

All our calculations are for the luminosity $L=1.3 \times 10^{46} \mathrm{erg} \mathrm{s}^{-1}$, which is the Eddington limit for a $1 n^{8} M_{0}$ black hole. Inder the condition of such a fixed luminosity, $Q \propto(\langle h v\rangle)^{-1}$ and the total mass $\left(M_{s}\right)$ associated with the shell is given, in terms of solar mass $\left(M_{0}\right)$, by

$$
\log \left(M_{S} / M_{0}\right)=2.31-\log (\langle h v\rangle)-\log \left(n_{H}\right)
$$

For example, with $\log [\langle h \nu(e r g)\rangle]=-8.73$ (corresponding to $\alpha=0.5$ ) and $\log$ $\left[n\left(\mathrm{~cm}^{-3}\right)\right]=10$, equation (9) yields $\log \left(M_{s} / M_{0}\right) \approx 1.0$. In the situation where the spectral transition between young quasar and quasar occurs at constant luminosity (i.e. at constant accretion rate; see paper I), the mass in the shell would remain invariant if the product $(\langle h \nu\rangle) \times n_{H}$ remains constant (see Equation (9)). The cases listed in Table 1 which correspond to such a 
situation are indicated as such.

As discussed by Ferland and Mushotzky (1982), the radiative acceleration due to photoionization of hydrogen in the gas shell is $\left(g_{\text {rad }}\right)_{j} \approx(1-4) \times 10^{-10}$ $\mathrm{T}_{4}^{-3 / 4} \mathrm{n}_{\mathrm{H}} \mathrm{cm} \mathrm{s}^{-2}$. For comparison, we note that the radiative acceleration due to electron scattering $\left(g_{r a d}\right)_{s}$ in a neutral plasma is given by

$$
\left(g_{\mathrm{rad}}\right)_{s}=\sigma_{0}\langle h v\rangle n_{v} / \mathrm{m}
$$

where $\sigma_{0}$ is the Thomson cross-section and $(m)$ is the mass of hydrogen. The ratio of these accelerations may be expressed as

$$
\frac{\left(g_{\text {rad }}\right)_{s}}{\left(g_{\text {rad }}\right)_{i}}=A \cup\left(T_{4}\right)^{3 / 4}\langle h \nu(k e V)\rangle
$$

where $A \approx 2-8$. We note that, for $T_{4} \geq 1$ and a flat continuum spectrum characterized by $\alpha \approx 0$, the radiative acceleration would be dominated by electron scattering when $U \geq 10^{-1}$. This situation is compatible with the Eddington limited regime that we associate with young quasars in that it allows for an ample mass supply to the accretion disk. ${ }^{2}$ In contrast, for typical quasars characterized by $\alpha=0.5-1, U \approx 10^{-2}$ and $T_{4} \approx 1$, we find (from equation $(11))$ that $\left(g_{\text {rad }}\right)_{s} \leq 10^{-1}\left(g_{r a d}\right)_{i}$. This latter condition is compatible with a quasar situation where BLR clouds could be radiatively driven away from the central source by photoionization even though electron scattering may no longer be sufficient to balance gravity (i.e. when the luminosity of a quasar is below the Eddington limit; see Paper I).

Table 1 summarizes the intensities calculated for some of the principal atomic emissions expected from shells of gas corresponding to broad line regions (BLR) and narrow line regions (NLR). We consider a fixed luminosity 
$\left(L=1.3 \times 10^{46} \mathrm{erg} \mathrm{s}^{-1}\right)$ and take as our independent variables the spectral energy index $(\alpha)$ of the central source of radiation, the hydrogen density $\left(n_{H}\right)$ characterizing the gas and the ionization parameter (U) that relates the density of primary photons to that of the gas. The Rydberg luminosity $\left(L_{R} \equiv\right.$ $\left.Q \times E_{R}\right)$ of the central source and the inner radius $(r)$ of the surrounding gas shell are derived quanties (via equation (5)), but are also listed to provide guide scales for the primary photon source strength and the size of the region of secondary emission. If we assume a black hole mass of $-1 n^{8} M_{0}$ for young quasars (see Paper I) and $\leq 10^{9} M_{0}$ for quasars (for the luminosity considered here) the BLR inner radius corresponds to $r \geq 10^{3} \mathrm{GM} / \mathrm{c}^{2}$ (see cases \#1-10), consistent with a region suitably larger than the accretion disks we associate with such objects.

Following Netzer, Wills and Wills (1982), we assume $U \approx 10-2$ is appropriate for evaluating the excitation of the NLR clouds of quasars as well as the BLR clouds and that the hydrogen density of such NLR gas is characterized by $n_{H} \approx\left(10^{3}-10^{4}\right) \mathrm{cm}^{-3}$. As shown in Table 1 (cases \#11-15), the NLR inner radius corresponds to $r \geq 10^{2} \mathrm{pc}$, comparable to a galactic structure scale. For both NLR and BLR shells, our specification of ionization parameter (U) yields $\log \mathrm{N}=21.75$ for quasars and $\log \mathrm{N}=21.75-22.75$ for young quasars (see eq. (7)), a regime where the electron scattering optical depth is substantially less than unity.

As shown in Table 1 (Cases \#1-4), we have calculated the BLR emissions to be expected from quasars (QSO) for a range of conditions corresponding to $\alpha=$ 0.5-1. 0 for the central source, $\log \left(n_{H} \mathrm{~cm}^{-3}\right)=9-11$ for the hydrogen number density in the shell and a fixed ionization parameter $U=10^{-2}$. In comparing these calculations to observations, we examine several line intensity ratios of particular interest; these are exhibited in Table 2. Considering the 
ranges in the calculated ratios and the ranges in the observed ratios for quasars, the agreement appears to be adequate for us to have confidence that our model may be used for evaluating the effects of the more extreme values in the basic parameters which characterize young quasars. In particular, we take $\alpha>0$ as a fundamental property of the spectrum for the central source of a young quasar (YOSO) and consider $U=10^{-1}$ as well as $U=10^{-2}$ (see Cases $\# 5-10,14,15$ in Table 1). By doing this it becomes evident that, in evolving from YQSO to QSO at constant luminosity (i.e. constant accretion rate) and constant cover fraction, most strong BLR emissions generally increase by an order of magnitude or more (e.g. hydrogen free-bound $n \geq 2$

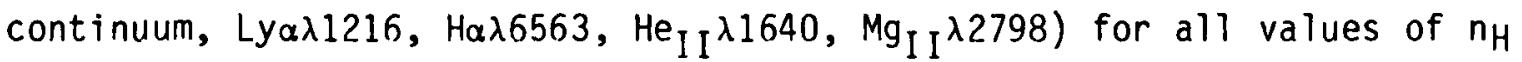
assumed. Furthermore, this pronounced trend persists when varying the ionization parameter for YOSOs from $U=10^{-1}$ to $U=10^{-2}$. However, $0_{V I} \lambda 1034$ would remain relatively constant when starting with YOSOS having $U=$ 10-1 (Cases \#8-10), a situation compatible with Eddington limited accretion. Contrary to the trend for most strong BLR lines, the NeVIII $\lambda 774$ emission from YQSOS could be substantially greater than from QSOS, provided that a decrease in the ionization parameter (and consequently shell thickness) is associated with the transition (i.e. cases \#8-10 compared with \#1-4). Similar pronounced behavior could also occur for NLR emission (e.g., compare case \#15 with $\# 11-13)$. While the absolute line intensities listed in Table 1 should be considered with caution (e.g. all are normalized to a cover fraction of unity), they are appropriate for the initial identification of such major trends. The possibility that the signature of YQSO line emissions could be substantially different from that of OSOS, independent of normalization problems, can be noted by comparing line ratios; two ratios of particular interest are listed in Table 3. 
IV. IMPLICATIONS OF SPECTRAL EVOLUTION ON SOURCE COUNTS

Based upon the continuum spectrum we have identified for the residual $C X B$ (Section II), we have shown that the dominant young quasar (YOSO) sources of this background would tend to be underluminous in many of their BLR optical line emissions by an order of magnitude or more relative to quasars (OSO) of comparable luminosity (Section III). In particular, the Lya $\lambda 12.16$ line emission from a YOSO would be well below that expected from a quasar of equal luminosity and RLR cover fraction (see Table 1). For quasar continua that bulge in the UV (e.g. due to a thermal component) this effect could easily exceed two orders of magnitude. By searching for redshifted Lya in the band 5700-6900 $\AA$ and finding no such sources, Osmer (1982) concludes that there is a paucity of Lya emitting quasars at $z>3.5$ relative to what might have been expected from extrapolations at lower redshift (also see Schmidt and Green 1983). As pointed out in Paper I, the dominant sources of the CXB are likely to be at these higher redshifts if they are indeed precursors of the AGN we are familiar with. In such a case the apparent deficit of typical quasars at such redshifts could be due to a bias against detection of young quasars and precursor active galaxies in any experiment that is based upon the assumption that Lya line intensity is an invariant measure (i.e. relative to redshift) of source luminosity. On the other hand, the possibility that the $0_{V I} \lambda 1034$ line intensity of young quasars might be comparable to that of like luminosity quasars could lead to an associated YQSO line in the 5700-6900 $\AA$ band, if these sources are at the appropriate redshifts (i.e. $z=4.5-5.7$ ). However, the $0_{V I} \lambda 1034$ line is not likely to have been observed at the sensitivity level of Osmer's particular survey since the emitted intensity of this line would be an order of magnitude below that of Lya emission from quasars of comparable luminosity 
(see Table 1).

The observation of the radio-loud quasar PKS 2000-330 at $z=3.78$

(Peterson, Savage, Jauncey and Wright 1982) as a strong source of redshifted

optical lines (including Lya $\lambda 1216$ and $\mathrm{V}_{\mathrm{I}} \lambda 1034$ ) indicates that "seeing"

effects (e.g. dust) can not readily account for the lack of quasars observed at such high redshift. As pointed out by Peterson et al. (1982), the surface density of radio OSOS is only - $8 \%$ of the surface density of optical QSOS, but the number of radio QSOs and optical QSOS with $z>3$ is comparable, with the two QSOS at highest redshift being radio loud. This may be an indication that high luminosity radio loud QSOS turn on sooner than most QSOS, possibly at about the same epoch as YOSOS. In order to detect YQSOs in the same $4200-8200$ \& band where Peterson et al. (1982) observed lines from PKS $2000-330$, it would be necessary to trigger on optical emission rather than radio since YOSOS are expected to be thermal sources (and therefore radio quiet; see Paper I). The OVI $\lambda 1034$ line could be appropriate for such a YOSO search since the flux, relative to $L y \alpha$, is likely to be an order of magnitude more than that expected from QSOS (see Table 3). At shorter wavelengths, the NeVIII $\lambda 774$ line from YOSOs could be observable at a level which, relative to Lya, is much higher than expected from QSOS. This neon line would thereby be a powerful optical means of recognizing YOSOs if they are indeed Eddington limited sources requiring a higher value for the ionization parameter (U) than that characterizing quasars. The UV continuum of such high redshift YQSOS would not be readily detectable, however, since such emission could well be two orders of magnitude below that of QSOS having comparable luminosity (see Figure 2).

Comparing the source counts at high redshifts for radio loud quasars, radio quiet quasars and young quasars (as spectrally defined in Section III) 
could provide the framework for understanding the early stages of quasar evolution (e.g. spectral versus luminosity evolution). A scheme based upon spectral evolution (Paper I) is to be distinguished from models involving obscuration by intergalactic dust as the explanationfor the paucity of quasars at high redshifts (Ostriker and Cowie 1981).

\section{v. CONCLUSIONS}

We propose that the apparent turn-over in the redshift distribution of usual quasars at $z \geq 3.5$ could be due to a situation in which the dominant sources of the CXB, including young quasars, are underluminous in Lya 1 ine emission. For a situation where these CXB sources correspond to emission near the Eddington luminosity limit, we suggest that the observation of $0_{V I} \lambda 1034$ and NeVII $\lambda 774$ lines, comparable in flux to Lya, would readily distinguish these objects from quasars. As pointed out in Paper I, we expect that the number of YQSOS would dominate quasars at $z \geq 3$. At still higher redshift $(z \geq 4)$, we expect that most compact sources would be precursor active galaxies (PAGs) of relatively short lifetime ( $10^{8}$ years). Since these PAGs would probably be formed early in the epoch of galaxy formation, elements such as oxygen and neon could have abundances much below their "solar" values. As such, spectral lines due to elements other than hydrogen and helium might not be useful for identifying these new objects. On the other hand, being the dominant sources of the CXB, these PAGs should exhibit remarkably flat X-ray spectra (below - $10 \mathrm{keV}$ ). The broadband X-ray telescope planned for the AXAF mission (Zombeck 1982) should thereby provide a suitable means for identifying such objects and distinguishing them from quasars. ${ }^{3}$ 


\section{ACKNOWLEDGMENTS}

We thank Richard Green for encouraging us to undertake these calculations, Gary Ferland for providing us with the appropriate computer code and to Richard Mushotzky for showing us how to run the programs. The possible importance of NeVIII $\mathrm{N}^{774}$ line emission was suggested by Gary Ferland.

\section{FOOTNOTE}

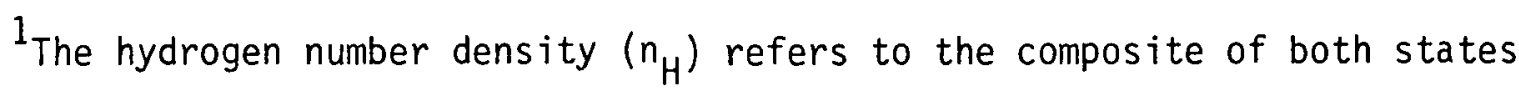
(ions and atoms).

${ }^{2}$ For young quasars with $U \gtrsim 10^{-1}$ and $T_{4}=1-10$, the gas pressure would be lower than that which may be required for supporting a multiphase medium (Guilbert, Fabian and McCray 1983).

\footnotetext{
3 Worrall and Marshall (1983) have determined that the average $X$-ray spectrum for a sample of QSOs of visual magnitude brighter than 16 requires a spectral index $\alpha>0.6$, over the band 1-15 keV.
} 


\section{REFERENCES}

Boldt, E. 1981, Comments Ap. 2, 97.

Condon, J.J., Jauncey, D.L., and Wright, A.E. 1978, A. J. $83,1036$.

Davidson, K. and Netzer, H. 1979, Rev. Mod. Physics 51, 715.

DeZotti, G., Boldt, E., Cavaliere, A., Danese, L., Franceschini, A., Marshall,

F., Swank, J., and Szymkowiak, A. 1982, Ap. J. 253, 47.

Ferland, G. and Mushotzky, R. 1982, Ap. J. 262, 564.

Ferland, G. and Truran, J. 1981, Ap. J. 244, 1022.

Green, R., Pier, J., Schmidt, M., Estabrook, F., Lane, A. and Wahlquist, H. 1980, Ap. J. 239, 483.

Guilbert, P., Fabian, A. and McCray, R. 1983, Ap. J. 266, 466.

Holt, S., Mushotzky, R., Becker, R., Boldt, E., Serlemitsos, P., Szymkowiak,

A. and White, N. 1980, Ap. J. 241, L13.

Kazanas, n. and Shafer, R. 1982, personal communication.

Kazanas, D. and Shafer, R. 1983, in "Positron-Electron Pairs in Astrophysics" (ed.

M. Burns, A. Harding and R. Ramaty), AIP, New York (in press).

Kwan, J. and Krolik, J. 1981, Ap. J. 250, 478.

Leiter, D. 1983, in "Positron-Eleciron Pairs in Astrophysics" (ed. M. Burns:

A. Harding and R. Ramaty), AIP, New York (in press).

Leiter, D. and Boldt, E. 198\%, Ap. J. 260, 1 (Paper I).

Malkan, M. and Sargent, W. 1982, Ap. J. 254, 22.

Marshall, F., Boldt, E., Holt, S., Miller, R., Mushotzky, R., Rose, L.A.,

Rothschild, R. and Serlemitsos, P. 1980, Ap. J. 235, 4.

Maxon, S. 1972, Phys. Rev. A $5,1630$.

Mckinley, J. and Ramaty, R. 1983, in preparation.

Mushotzky, R. 1982, Ap. J. 256, 92.

Netzer, H. Wills, B.J. and Wills, D. 1982, Ap. J. 254, 489.

Dstriker, J.P. and Cowie, L.L. 1981, Ap. J. 243, L127. 
Osmer, P.S. 1982, Ap. J. 253, 28.

Peterson, B., Savage, A., Jauncey, D. and Wright, A. 1982, Ap. J. 260, L27.

Petre, R., Mushotzky, R., Krolik, J. and Holt, S. 1982, Bull AAS 14, 962.

Pozdnyakov, L., Sobol, I. and Syunyaev, R. 1977, Sov. Astron. 21(6), 708; Astron. Zh. 54, 1246.

Ramaty, R. and Meszaros, P. 1981, Ap. J. 250, 384.

Rothschild, R., Mushotzky, R., Baity, W., Gruber, D., Matteson, J. and Peterson, L. 1983, Ap. J. 269, 423.

Schmidt, M. and Green, R. 1983, Ap. J. 269, 352.

Worral1, D. and Marshal1, F. 1983, Ap. J. (submitted).

Zombeck, M.V. 1982, presented at Symposium on Advanced Space Instrumentation in Astronomy, XXIV COSPAR (Ottawa, Canada), to appear in COSPAR Proceedings. 
TABLE 1: Optical Radiation To Be Observed From A Shell Photoexcited By A Central Source.

$L\left(13.6-10^{5} \mathrm{eV}\right)=1.3 \times 10^{46}$ ergs $\mathrm{s}^{-1}$

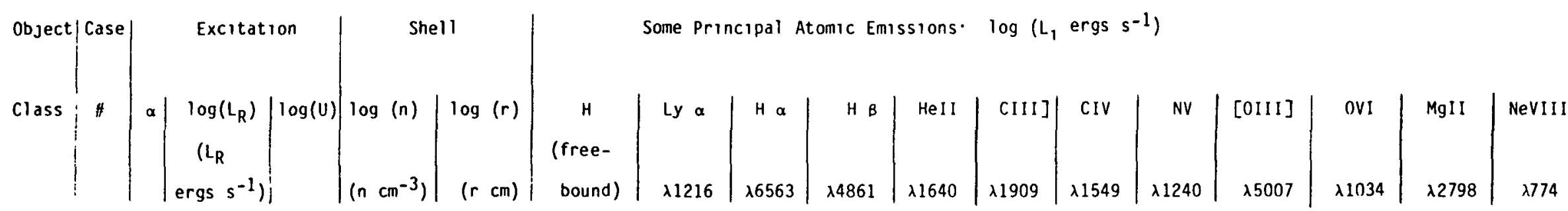

\begin{tabular}{|c|c|c|c|c|c|c|c|c|c|c|c|c|c|c|c|c|c|c|}
\hline & 1 & 1 & 45.17 & -2 & 10 & 18.13 & 44.09 & 45.13 & 44.55 & 43.78 & 43.98 & 44.23 & 45.07 & 43.81 & 40.96 & 44.00 & 44.44 & 40.81 \\
\hline OSO & $2^{a}$ & $1 / 2$ & 44.18 & -2 & 10 & 17.64 & 43.34 & 44.43 & 43.84 & 43.46 & 43.17 & 43.57 & 44.28 & 43.21 & 40.13 & 43.64 & 43.24 & 41.25 \\
\hline (BLR) & $3^{b}$ & $1 / 2$ & 44.18 & -2 & 11 & 17.14 & 43.58 & 44.09 & 43.32 & 43.13 & 43.09 & 43.84 & 44.37 & 43.60 & 39.51 & 43.93 & 43.34 & 41.60 \\
\hline & 4 & $1 / 2$ & 44.18 & -2 & 9 & 18.14 & 43.06 & 44.59 & 43.64 & 42.20 & 43.12 & 43.81 & 44.05 & 43.03 & 40.61 & 43.53 & 43.36 & 41.16 \\
\hline
\end{tabular}

\begin{tabular}{|c|c|c|c|c|c|c|c|c|c|c|c|c|c|c|c|c|c|c|}
\hline & $1^{5}$ & 0 & 43.20 & -2 & 9 & 17.65 & 42.07 & 43.76 & 42.98 & 41.51 & 41.94 & 42.99 & 42.67 & 41.61 & 39.32 & 42.18 & 42.40 & 40.05 \\
\hline & 6 & 0 & 43.20 & -2 & 10 & 17.15 & 42.57 & 43.62 & 42.99 & 42.78 & 41.98 & 42.97 & 43.30 & 42.19 & 39.36 & 42.55 & 42.64 & 40.24 \\
\hline YQSO & 7 & 0 & 43.20 & -2 & 11 & 16.65 & 42.69 & 43.16 & 42.38 & 42.24 & 42.00 & 42.20 & 43.55 & 42.92 & 38.48 & 43.33 & 41.34 & 41.37 \\
\hline \multirow[t]{3}{*}{ (BLR) } & $8^{\mathrm{a}}$ & 0 & 43.20 & -1 & 9 & 17.15 & 42.69 & 43.44 & 42.36 & 41.65 & 42.11 & 43.34 & 43.27 & 42.82 & 40.20 & 43.77 & 38.30 & 43.91 \\
\hline & $9^{b}$ & 0 & 43.20 & -1 & 10 & 16.65 & 42.68 & 43.36 & 42.20 & 41.93 & 42.12 & 42.72 & 43.97 & 42.97 & 38.98 & 43.84 & 37.70 & 43.92 \\
\hline & 110 & 0 & 43.20 & -1 & 11 & 16.15 & 42.76 & 43.16 & 41.82 & 41.77 & 42.09 & 42.40 & 43.39 & 43.10 & 38.41 & 43.82 & 38.24 & 43.92 \\
\hline QSO & 11 & 1 & 45.17 & -2 & 4 & 21.13 & 43.90 & 45.40 & 44.24 & 43.43 & 43.98 & 44.30 & 44.71 & 43.50 & 44.94 & 43.73 & 43.71 & 40.46 \\
\hline \multirow[t]{2}{*}{ (NLR) } & $12^{c}$ & $1 / 2$ & 44.18 & -2 & 4 & 20.64 & 42.94 & 44.71 & 43.41 & 42.23 & 43.12 & 43.69 & 43.92 & 42.91 & 43.72 & 43.41 & 42.77 & 41.03 \\
\hline & 13 & $1 / 2$ & 44.18 & -2 & 3 & 21.14 & 42.94 & 44.74 & 43.46 & 42.43 & 43.12 & 43.66 & 43.91 & 42.90 & 43.72 & 43.40 & 42.71 & 41.02 \\
\hline
\end{tabular}

\begin{tabular}{|c|c|c|c|c|c|c|c|c|c|c|c|c|c|c|c|c|c|}
\hline YOSO & 140 & 43.20 & -2 & 3 & 20.65 & 41.80 & 43.91 & 42.49 & 41.40 & 41.92 & 42.83 & 42.52 & 41.44 & 42.35 & 42.01 & 41.86 & 39.88 \\
\hline (NLR) & $15^{c} \quad 0$ & 43.20 & -1 & 3 & 20.15 & 42.79 & 43.69 & 42.58 & 42.11 & 42.11 & 43.32 & 43.79 & 42.73 & 43.64 & 43.73 & ו7.79 & 3.8 \\
\hline
\end{tabular}

\footnotetext{
${ }^{a}$ Constant mass in shell $=11.3 \mathrm{M}_{0}$

${ }^{b}$ Constant mass in shell $=1.19 \mathrm{M}_{0}$

c Constant mass in shell $=1.13 \times 10^{7} \mathrm{M}_{0}$
} 


\section{TABLE 2: Comparison With QSO Dbservations}

Ratio

$$
L y \alpha / H \beta
$$

$H_{\alpha} / H_{\beta}$

C IV $_{\text {I1549/Lya }}$

$\left.C_{\text {III }}\right] \times 1909 / C_{I V^{\lambda 1549}}$

$0.14-0.58$

$0.29-1.9$

$0.15-0.64$

$N_{V} \lambda 1240 /$ Lya

$0_{V I \lambda 1034 / L y \alpha}$

Mg II $\lambda 2798 / \mathrm{HB}$

$0.60-14$

$0.05-0.32$

$0.07-0.69$

$0.10-0.35$

${ }^{\mathrm{a}}$ From Table 1, Cases \#1 - \#4

brom Table 6 by Kwan and Krolik (1981)

${ }^{c}$ Intrinsic intensity ratio for 3 C351, after reddening correction (Netzer, Wills and Wills, 1982) 
TABLE 3: YQSO Comparison with QSO

\begin{tabular}{|c|c|c|}
\hline $\begin{array}{l}\text { Ratio } \\
\text { (RLR) }\end{array}$ & $\begin{array}{c}\text { Calculated Range } \\
\text { (QSO) }\end{array}$ & $\begin{array}{c}\text { Calculated Range } \\
\text { (YQSO) }\end{array}$ \\
\hline $0_{V I} \lambda 1034 / L y_{\alpha}$ & $0.07-0.69$ & $2.1-4.6$ \\
\hline $\mathrm{Ne}_{V I I I} \lambda 774 / L \mathrm{y} \alpha$ & $<0.003$ & $3.0-5.8$ \\
\hline aFrom Table 1, Cases \#1 - \#4 & & \\
\hline
\end{tabular}


Figure 1 - The spectral density of the estimated residual CXB (Leiter and Boldt 1982), relative to the total CXB observed with HEAO-1, is plotted as a function of photon energy, in kev. Statistical errors are indicated for those data points where they exceed the size of the dots.

Figure 2 - The total number of photons (up to $100 \mathrm{keV}$ ) emitted per second $Q(>E)$ by the central source of continuum radiation is plotted as a function of the energy lower-limit (E) for the integration. The curves shown correspond to underlying power-law energy spectra of index $\alpha=0$ and $\alpha=1$, as indicated. Both curves correspond to a luminosity $L=10^{46}$ erg $\mathrm{s}^{-1}$ over the band $13.6 \mathrm{eV}-100 \mathrm{keV}$. The band of energies corresponding to an optical depth greater than unity $(\tau>1)$ is shown for a shell thickness of $0.1 \mathrm{~g} \mathrm{~cm}^{-2}$. 


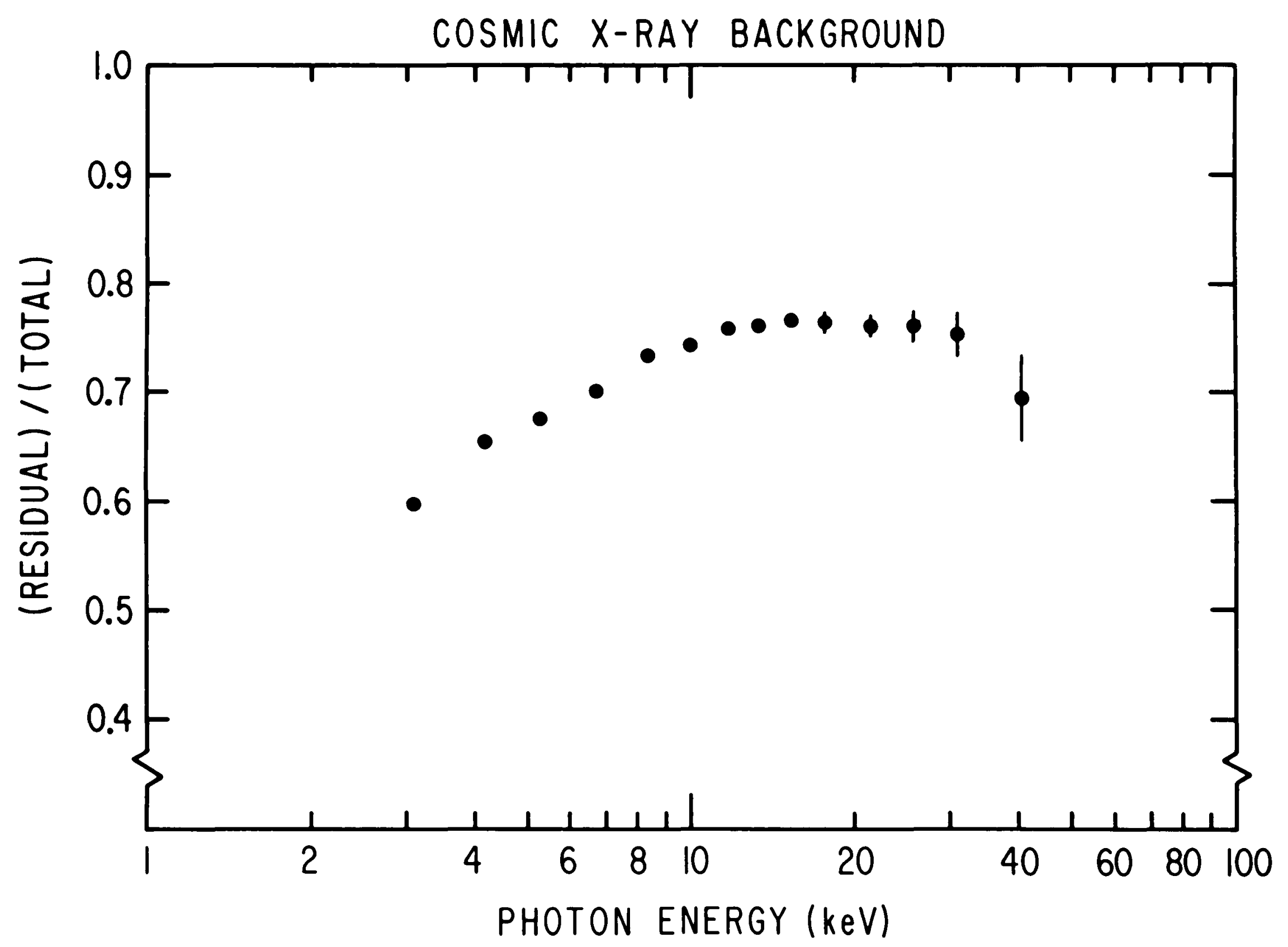

Figure 1 
SOURCE STRENGTH [Q(>E)] FOR IONIZING PHOTONS

OF ENERGY EXCEEDING (E)

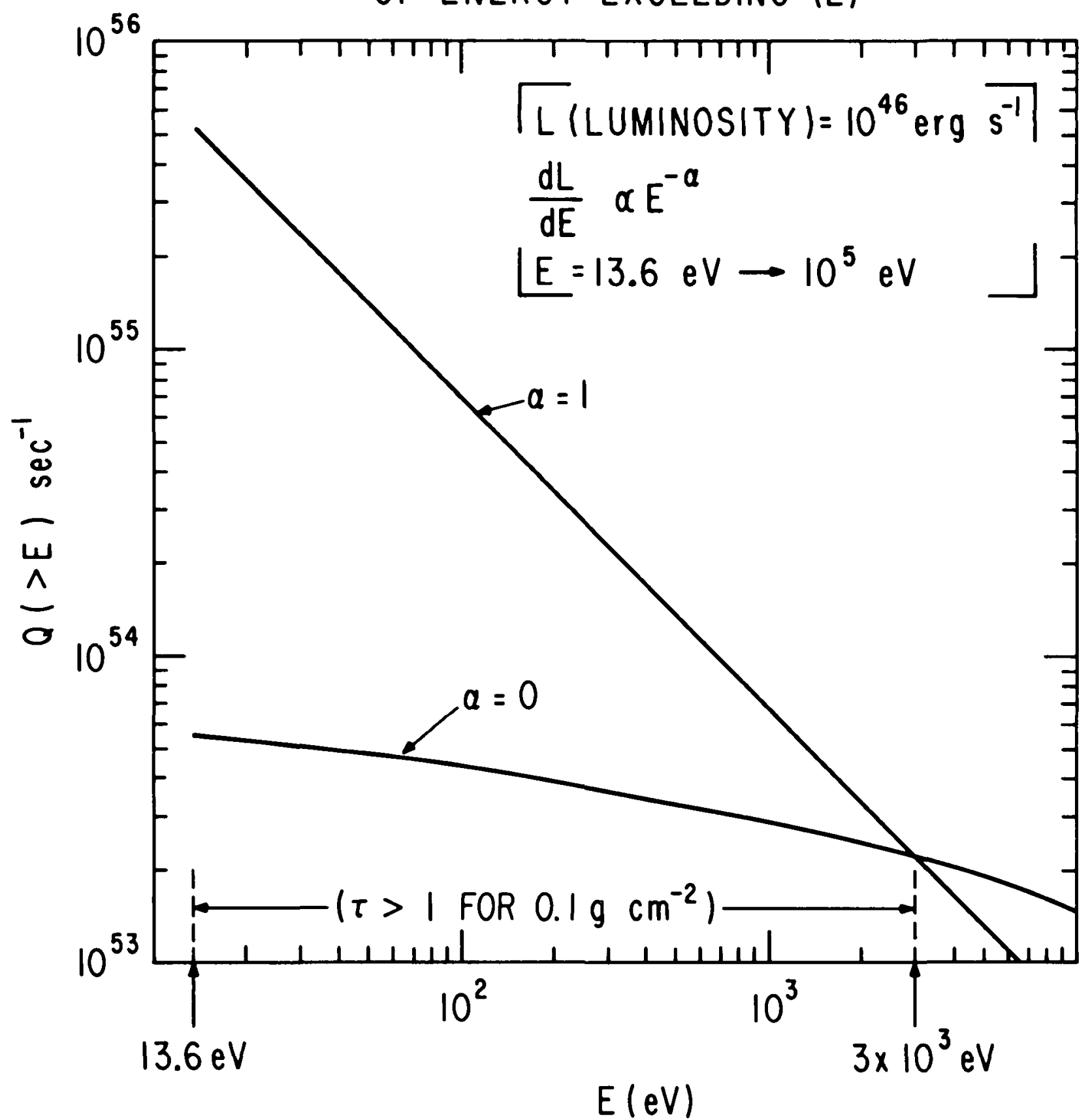

Figure 2 
ADDRESS OF AUTHORS

ELIHU A. BOLDT, Code 661, Laboratory for High Energy Astrophysics, NASA/ Goddard Space Flight Center, Greenbelt, MD 20771 and DARRYL LEITER, Sciences Div., FSTC, Charlottesville, VA 22901 
BIBLIOGRAPHIC DATA SHEET

\begin{tabular}{|c|c|}
\hline 2 Government Accession No. & 3. Recipient's Catalog No. \\
\hline 4. Title and Subtitle & $\begin{array}{l}\text { 5. Report Date } \\
\text { July } 1983\end{array}$ \\
\hline AS SOURCES OF THE COSMIC X-RAY BACKGROUN & $\begin{array}{l}\text { B. Performıng Organızation Code } \\
661\end{array}$ \\
\hline $\begin{array}{l}\text { 7. Author(s) } \\
\text { Elihu Boldt and Darryl Leiter }\end{array}$ & 8. Performıng Organızatıon Report No. \\
\hline $\begin{array}{l}\text { 9. Performing Organization Name and Address } \\
\text { Code } 661\end{array}$ & 10. Work Unit No. \\
\hline $\begin{array}{l}\text { Lab. for High Energy Astrophysics } \\
\text { NASA/Goddard Space Flight Center } \\
\text { Greenbelt, MD } 20771\end{array}$ & 11. Contract or Grant No. \\
\hline \multirow[t]{2}{*}{ 12. Sponsoring Agency Name and Address } & \\
\hline & 14. Sponsorıng Agency Code \\
\hline
\end{tabular}

15. Supplementary Notes

Accepted for publication in The Astrophysical Journal.

\section{Abstract}

The sources which dominate the thermal cosmic X-ray background cannot have $X-r a y$ spectra similar to the power laws measured for bright active galactic nuclei. We pursue the optical consequences of this disparity by considering a standard model for the photoexcitation and heating of the line emitting gas surrounding a central source (e.g. such as a quasar). The optical line emission to be associated with compact young quasar sources having the same $X-r a y$ spectrum as the $x$-ray background is found to be substantially different from that characteristic of typical quasars. Implications on quasar source counts and the identification of such new objects are discussed.

17. Key Words (Selected by Author(s)) cosmology - galaxies: nuclei - quasars 18. Distribution Statement

X-rays: sources - X-rays: spectra

\begin{tabular}{|l|l|} 
19. Security Classif. (of this report) & 20. Security Classif. (of this page)
\end{tabular} $\mathrm{U}$ $\mathrm{U}$ 21. No. of Pages 22. Price 23 